\title{
$\mathrm{XX}$.
}

\section{Über ärztliche Fürsorge für Taubstumme nebst Vorschlägen zur Reorganisation des Taubstummenbildungswesens.}

Von

Professor 0stmann in Marburg.

In einer Zeit, in der die soziale Fürsorge mebr wie je Strat und Gesellschaft beschäftigt, kann es nicht Wunder nehmen, daß der Fürsorge für die Taubstummen und ihrer Fortbildung ein erhöhtes Interesse sich zuwendet, und insbesondere auch diejenigen ärztlichen Kreise erfaßt, welche sich die Erkrankungen des Ohres und der Grenzgebiete zu ihrem besonderen Arbeitsfeld erkoren haben.

Bis zu der Zeit, wo man mit Hilfe einer geeigneten Lehrmethode die Bildungsfähigkeit der Taubstummen nachweisen konnte, kann von einer allgemeinen Fürsorge für dieselben nicht gesprochen worden; die Taubstummen wurden vor dem Gesetz den Blödsinnigen und Unmündigen gleich geachtet.

Mit dem methodischen Unterricht der Taustummen-Kinder war der erste große Sehritt allgemeinerer Fürsorge getan. Politzer hat in seiner vortrefflichen Geschichte der Obrenheilkunde 1) den Stand des Taubstummenunterrichts bis zum Ende des 18. Jahrhunderts und das Wirken derjenigen Männer geschildert, welche in aufopfernder Arbeit sich um die Förderung des Unterrichts und damit um die Hebung des geistigen Niveans wie der bürgerlichen Stellung der Taubstummen innerhalb dieses Zeitraums verdient gemacht haben.

Mit Heine ek e, welcher 1778 in Leipzig die erste Taubstummenanstalt gründete, beginnt in Deutschland ein zielbewußter Taubstummenunterricht.

1) Bd. I S. 427 u. f. 
Mehr als ein Jahrhundert ist seit Heineckes Tode - im Jahre 1790 - verflossen, und trotz dieser langen Zeit, in welcher die Bildungsfähigkeit der Taubstummen voll erwiesen und von dem Gesetzgeber durch die veränderte Stellungnahme ihnen gegenüber anerkannt worden ist, ${ }^{1}$ ) mußte bei der VerhandInng ïber das Taubstummen-Bildungswesen der Abgeordnete Rzesnitzek im PreuBischen Abgeordnetenhause feststellen, ${ }^{2}$ ) daß in den Taubstummenanstalten Schlesiens zu Ostern 1900 ungefähr 600 Schüler aufgenommen werden konnten, während etwa 500 unbeschult blieben. Da die Verhältnisse in anderen Provinzen wahrscheinlich ähnlich liegen, so bedarf es auf dem Gebiete der Beschulung unzweifelhaft einer verstärkten Fürsorge; denn es ist nicht einzusehen, weshalb gerade diejenigen Kinder, welche durch ein unheilbares Leiden in der schwersten Weise in ihrer Arbeitsund Erwerbsfähigkeit geschädigt worden sind, auch noch in dem Anspruch auf die Möglichkeit der Schulbildung hinter den vollsinnigen Kindern zuückstehen sollen. Wenn sich dieser Fürsorge dadurch Schwierigkeiten in den Weg stellen, daB die taubstummen Kinder untereinander hinsichtlich ihrer Bildungsfähigkeit vielleicht ungleichwertiger sind als die vollsinnigen, schulpflichtigen Kinder, so lassen sich doch die Schwierigkeiten mit wenig veränderten Einrichtungen und bei mäßiger Erhöhung der zur Zeit für das Taubstummenbildungswesen aufgewandten Mittel wohl überwinden.

Die Bereitstellung weiterer Mittel wird vielleicht auch dadurch erschwert, daß der Fernerstehende so wenig offensichtlichen Erfolg von der Aufwendung dieser Mittel sieht. Dieser Eindruck entspricht indes nicht den Tatsachen; denn wenn man den Eindruck, welchen taubstumme Kinder bei ihrem Eintritt und Austritt aus der Taubstummenschule machen, mit einander vergleicht, und in jahrelanger Beobachtung die ebenso mühevolle wie segensreiche, stille Arbeit der Taubstummenlehrer verfolgt, so kann man vom rein menschlichen wie vom ärztlichen Standpunkt aus nur dringend wünschen, daß einem jeden taubstummen Kinde, sofern es sich überhaupt als bildungsfähig erweist, die Möglichkeit der Beschulung geboten wird.

Aber für einen jeden, der nicht das taubstumme Kind in seiner gesammten körperlichen wie geistigen Entwicklung betrachtet,

1) P. Schlotter, Die Rechtsstellung und der Rechtsschutz der Taubstummen; Blätter für Taubstummenbildung XX. Jahrg. No. 8 S. 121. 1907.

2) Blätter für Taubstummenbildung XX. Jhrg. No. 8. 1907 S. 121. Stenographischer Bericht der Verhandlungen. 
muB in nicht wenigen Fällen der Erfolg unserer heutigen Unterrichtsmethode als wenig befriedigend erscheinen, insbesondere toweit es sich um Verstehen und Verstandenwerden im Ver Juhr mit den Mitmensehen nach dem Verlassen der Anstalt $\$$ handelt.

Es ist in den letzten Jahren von ärztlicher Sge'mehrfach auf den unbefriedigenden Erfolg des Unterrichts getare nach dieser Richtung hingewiesen worden, und es kann ant nach meinen Beobachtungen kein Zweifel bestehen, daß bei nicht wenigen Kindern nach der Entlassung aus der Taubstummenanstalt an die Stelle der mit unendlicher Mühe gelehrten und erlernten Sprache bald wieder die Gebärdesprache tritt.

Mit diesen Hinweisen, so möchte ich glauben, wünscht keiner einen Vorwurf gegen die Taubstummenlehrer und ihre Arbeitsfreudigkeit auszusprechen; es wird von ärztlicher Seite im wesentlichen nur das behauptet, was ein bekannter Taubstummenlehrer, A. Gutzmann, zu seinem Bedauern selbst zugeben muß, daß nicht „der Hälfte aller entlassenen Taubstummen Verstehen und Verstandenwerden im mündlichem Verkehr mit ihren hörenden Mitmenschen in genügendem Grade eignet." 1)

Auf Grund dieser Tatsachen den hohen Wert der deutschen Unterrichtsmethode an sich in Zweifel ziehen zu wollen, wäre dunrichtig; denn m. E. verfügen wir über keine andere Methode, durch welche in gleicher oder besserer Weise die geistige Durchbildung des taubstummen Kindes gefördert werden könnte. Die Methode paßt nur nicht für alle Kinder; so in erster Linie nicht für diejenigen, welche so geringe geistige Anlagen besitzen, daß sie kaưm als bildungsfähig bezeichnet werden können. Nach der andern Richtung darf man nun aber auch nicht von vornherein der Ansicht sein, daß die Methode sich nicht vielleicht für eine Minderheit der Kinder noch fruchtbringender als bisher gestalten liebe, indem man durch eine volle, methodische Auswertung ev. noch vorhandener Hörreste die Sprachentwicklung fördert.

Aus diesen Gesichtspunkten ergibt sich eine Dreiteilung der Taubstummen und somit auch der Taubstummenanstalten.

Von einer solchen sachgemäßen Scheidung der Taubstummen sind wir leider noch weit entfernt; es sind zunächst ganz vereinzelte Anfänge zur Bildung besonderer Hörklassen gemacht.

Eine Trennung der Hörlosen und der mit Hörresten versehenen Taubstummen ist aber sofort auf den energischen Wider-

1) Vor- und Fortbildung der Taubstummen. In zwanglosen Heften herausgegeben von A. Gutzmann, Berlin 1899. Heft 1, S. 15. 
stand nicht weniger, alt erfahrener Taubstummenlehrer gestoßen, da sich nach ihrer Ansicht nicht eine Scheidung der Taubstummen nach Hörresten, sondern allein nach der geistigen Begabung empfiehlt.

Meines Erachtens ist dieser letztere Standpunkt und eine ibm entsprechende Gruppierung der Taubstummen in 3 Bildungsstufen der richtigere; denn diese Einteilung läßt eine dem eigentlichen Wesen des Unterrichts sehr viel mehr entsprechende, einheitlichere Zusammenstellung des Kindermaterials zu.

Auch ist die Zahl der mit wirklich verwertbaren Hörresten versehenen Kinder im Allgemeinen viel zu klein, als daß sich bei der jetzigen Organisation des Taubstummenwesens in den einzelnen Anstalten eine Zusammenstellung von Hörklassen mit annähernd gleichaltrigen und somit gleich vorgebildeten Kindern ermöglichen ließe. An einzelnen Stellen ist dann auch verhältnismäßig schnell der Versuch des Hörunterrichts wieder aufgegeben worden.

Auf der 13. Versammlung der deutschen otologischen Gesellschaft zu Berlin im Jahre 1904') hat Denker als wichtiges Ergebnis einer im Bayrischen Kultusministerium März 1904 stattgehabten Konferenz zur Beratung der weiteren Ausbildung des Taubstummenunterrichts mitgeteilt, „daß überall da, wo die nötigen Mittel bereit gestellt werden können, die in den einzelnen Klassen vorhandenen, partiell Hörenden, sowie die später ertaubten und noch Sprachreste besitzenden Zöglinge von den übrigen getrennt in eigenen Klassen vereinigt und hier unter Anwendung einer Methode, welche bei den ersteren Auge und Ohr gleichzeitg in Anspruch nimmt, unterrichtet werden.

Denker erhoffte eine schnelle und befriedigende Lösung der Geldfrage und sah in diesem Beschluß „einen glänzenden Sieg der seit langen Jahren auf dieses Ziel gerichteten Bestrebungen Bezolds und seiner Freunde," gab der Überzeugung Ausdruck, dab mit diesem Beschlub ein hochbedeutsamer Schritt in der Entwicklung des Taubstummen-Unterrichtswesens getan sei und sprach den Wunsch aus, daß dem Beispiele Bayerns bald die übrigen Bundesstaaten folgen möchten.

Ich vermag nicht anzugeben, wie sich für Bayern bis jetzt die Geldfrage gelöst hat; ich kann nur mitteilen, wie weit die übrigen deutschen Bundesstaaten der Anregung Bayerns gefolgt sind.

1) Verhandlungen $S .72$. 
Auf Grund des nicht ganz zutreffenden Verzeichnisses der deutsehen Taubstummenanstalten in dem Lehrbuch von Koerner habe ich sämtliche deutsche Taubstummenanstalten mit Ausnahme derjenigen in München gebeten, mir über die Fragen Auskunft zu erteilen:

1, Werden die neu aufgenommenen Kinder mit der kontinuirlichen Tonreihe von einem Ohrenarzt untersucht; und

2. findet ein Sonder-Unterricht - Hörunterricht - der mit Hörresten versehenen Kinder statt?

Das Ergebnis dieser Umfrage dürfte nicht den von dieser oder jener Seite gehegten Erwartungen ganz entsprechen.

Von 78 Anstalten beantworteten die erste Frage 56 Anstalten mit "nein"; 18 fast ausschließlich süddeutsche Anstalten mit „ja"; 4. Anstalten dahin, daß eine derartige Untersuchung in beschränktem Umfange von den Lehrern ansgeführt werde. In einzelnen Anstalten war die Stimmgabelprüfung früher ausgeführt aber auf-gegeben worden, weil sich dieselbe nach dem Urteil der Lehrer für den Unterrieht und seine Gestaltung als wertlos gezeigt hatte.

Die zweite Frage, ob ein getrennter Uuterricht der mit Hörresten versehenen Kinder - sog. Hörunterricht - stattfinde, beantworteten 70 Anstalten mit „nein"; darunter mehrere aus Bayern.

In einer mitteldeutschen Anstalt wird, so weit möglich, die Bildung von Sonderklassen angestrebt; in einer norddeutschen Anstalt wurde der Hörunterricht nach mehrjährigen Versuchen als undurchführbar und unzweckmäBig aufgegeben, weil die Trennung der Kinder nach ihrer geistigen Veranlagung bessere Unterrichtsresultate ergab; in einer dritten Anstalt befand man sich noch im Stadium des an sich wenig aussichtsreichen Versuches. 5 Anstalten endlich beantworteten die Frage mit , ja $\mathrm{a}^{\text {" }}$; doch muß ich dahingestellt sein lassen, $o b$ in den Hörklassen dieser Anstalten der Unterricht ausschließlich ein sog. Hörunterricht ist.

Es ist somit die Zahl derjenigen Anstalten, welche sich bisher überhaupt nur zu einem Versuch entschlossen haben, sehr klein und aus der Stimmung, die die Verhandlungen der Taubstummenlehrer auf ihren Versammlungen z. Teil erkennen lassen, wie aus den mir unaufgefordert zugegangenen Äußerungen möchte ich entnehmen, daß nach dem Abflauen der ersten Begeisterung sich eine immer stärker werdende Gegenströmung in den Kreisen der Taubstummenlehrer gegen die von vielen derselben nicht als neu anerkannte Methode des sog. Hörunterriehts geltend machen dürfte. 
Diese Gegenströmung wird durch fehlgeschlagene Erwartungen und durch die in langjähriger, praktischer Erfahrung gewonnene Überzeugung, daß man die Kinder nach ihrer geistigen Begabung trennen müsse, sowie durch die betrübende Beobachtung getragen, daß die Frage des Hörunterrichts keine rein wissenschaftliche Frage mehr sei, sondern z. T. egoistischen Zweeken diene. Wenn ich mich nicht verpflichtet fühlte, gegenüber den freimütigen Äußerungen, die mir zugegangen sind, die strengste Diskretion zu wabren, so würde ich eigenartige Mitteilungen $\mathrm{zu}$ machen in der Lage sein.

Gegenüber einer solchen Stimmung bei einem, wie mir scheinen will, großen Teil der Taubstummenlehrer wird es vor allem richtig: sein, wenn anders der gute und gesunde Kern der Bezold'schen Anregung nicht wieder verloren gehen soll, die Frage von jedem Strebertum frei $\mathrm{zu}$ halten, und das Taubstummenbildungswesen so $\mathrm{zu}$ reorganisıeren, dab die Vereinigung gleichaltriger Kinder mit verwertbaren Hörresten zu Hörklassen unter prinzipiellem Festhalten der Teilung der Taubstummen nach ihrer geistigen Begabung möglich wird.

Die Schwierigkeiten, welche sich einer entsprechenden Reorganisation des Taubstummen-Bildungswesens entgegen stellen, liegen auf administrativem und finanziellem Gebiet; doch dürften dieselben, sobald die Notwendigkeit der Reorganisation erst erkannt ist, keineswegs schwer zu beseitigen sein.

Meine nachstehenden Ausführungen beziehen sich wesentlich auf Preußen, über dessen Taubstummenfürsorge ich einen gewissen Überblick habe.

Die Beschulung der Taubstummen ist Sache der Provinzialbehörden; es gibt nur ganz vereinzelte Anstalten, welche vom Staat, von Städten oder durch milde Stiftungen neben eigenem Erwerb oder Subventionen unterhalten werden. Es fehlt also eine einheitliche Zentralinstanz in engerem Sinne, was seine Nachteile aber auch den Vorteil hat, dah jede Provinz für sich oder im Zusammenschluß mit anderen die m. E. notwendige Reorganisation des Taubstummenbildungswesens durchführen kann.

Diese Reorganisation muß folgenden Forderungen gerecht werden:

1. Für jedes taubstumme Kind muß die Möglichkeit der Schulbildung gegeben sein.

2. Sämtliche taubstumme Kinder sind, sofern nicht Idiotie und damit völlige Bildungsunfähigkeit klar zu Tage liegt, nach vollendetem 6. Lebensjahr in Taubstummenvorschulen aufzunehmen, 
welche in zwei Vorklassen gegliedert engsten AnschluB an die Taubstummenschulen haben und nach dem Vorbild der Berliner Taubstummenvorschule 1) nach Art der Kindergärten ausgebildet werden.

3. In den Taubstummenvorschulen verbleiben die Kinder 2 Jahr bezw. nur so lange, bis sich ein sicheres Urteil über ibre Bildungsfähigkeit in den Taubstummenschulen nach Maßgabe des Lebrplans derselben abgeben läßt. Bei dieser Beurteilung haben Lehrer, Pflegerin und Arzt zusammenzuwirken. Diejenigen Kinder, welche sich als völlig bildungsunfähig erweisen, werden einer Anstalt für idiotische und schwachsinnige Kinder überwiesen oder in die häusliche Privatpflege zurückgegeben. Letzteres geschieht auch bei Kindern, deren ständiges Zusammenleben mit anderen taubstummen Kindern infolge körperlicher oder geistiger Gebrechen unzweckmäßig erscheint.

4. Die durch 2 jährige Beobachtung als bildungsfähig erkannten Taubstummen werden nach ihrer geistigen Begabung in 3 Gruppen geteilt, die ich als schwach (I. Gruppe), mittel (II. Gruppe) und gut (III. Gruppe) bildungsfähig bezeichne.

5. Dieser Gruppierung der Kinder in 3 Gruppen entspricht eine Dreiteilung der Taubstummenschulen je nach dem Lehrplan und der vorzugsweise angewandten Unterrichtsmethode.

Die erste Gruppe der Anstalten nimmt nach Beendigung der Vorschulklassen diejenigen Taubstummenzöglinge auf, welche als "schwach bildungsfähig" voraussichtlich nicht dazu gelangen, mit Hilfe der deutschen Unterrichtsmethode zu einer Lautsprache zu kommen, die einen mündlichen Verkehr in genügendem Grade mit einiger Sicherheit erwarten läßt. In diesen Anstalten tritt anstelle des Artikulationsunterrichtes die französiche Unterrichtsmethode, und es wird eine möglichst frühzeitige Heranbildung der Kinder zu handwerksmäßigen Arbeiten, insbesondere zur Feldund Gartenarbeit angestrebt. Sofern die Kinder nach vollendeten Schuljahren zum selbständigen Erwerb nicht fähig und zu ihrer Unterhaltung verpflichtete Dritte nicht vorhanden sind, verbleiben sie in Heimstätten, welche diesen oder einigen dieser Taubstummenanstalten angegliedert sind, oder sie werden in Taubstummenheime überführt, sofern solche von den Provinzialvereinen zur Unterstiitzung und Pflege von Taubstummen errichtet sind.

1) Gutzmann, Vor- und Fortbildung der Taubstummen. 2. Heft. 
Die zweite Gruppe von Anstalten nimmt die "mittel begabten" Kinder auf, also den weitaus größten Teil der jetzt in unseren Taubstummenanstalten befindlichen Zöglinge. In diesen Anstalten wird die deutsehe Unterrichtsmethode in der bisherigen Weise dem Unterricht zu Grunde gelegt. Die bereits bisher bestehende Fürsorge für die schulentlassenen Zöglinge in materieller, geistiger und seelsorgerischer Hinsicht 1 ) wird in den bisherigen Bahnen fort entwickelt und in soweit ergänzt, als ein systematischer Fortbildungsunterricht eingeführt wird, worüber an späterer Stelle zu handeln sein wird.

Die dritte Gruppe der Anstalten nimmt die besonders gut veranlagten Kinder auf; unter diesen haben wieder diejenigen den Vorzug, welche später ertaubt noch gute Sprachreste besitzen oder Hörreste aufweisen, welche eine wesentliche Förderung der Sprachentwickelung bei systematischer Auswertung dieser Hörreste erwarten lassen. In diesen Anstalten wird in Sonderstunden der partiell Hörenden dem Hörunterricht eine besondere Sorgfalt zugewandt, um die Sprachentwicklung der Kinder zu fördern. Dem gemeinsamen Unterricht liegt die deutsche Unterrichtsmethode zu Grunde.

Auf die zweite und dritte Gruppe der Taubstummenschulen baut sich die Fortbild ung s s ch ule auf, welche für einen gröberen Bezirk an eine oder zwei regionär günstig gelegene Taubstummenschulen angeschlossen wird. Thr Besuch ist fakultativ.

Der Lebrplan dieser Fortbildungsschulen lehnt sich eng an denjenigen der schon jetzt ganz vereinzelt bestehenden Fortbildungsschulen an, erfährt indes nach der Seite der sprachlichen Fortentwicklung der Zöglinge eine Erweiterung.

Der Sprung von der Taubstummenanstalt, zumal wenn es sich um ein Internat handelt, in das Getriebe des täglichen Lebens, von dem Verkehr mit dem Lehrer zu dem Umgang mit den verschiedensten Personen ist ein so ungeheurer, dab sehr viele Kinder sich nicht zurecht finden, im Gefiuhl ihrer sprachlichen Hilflosigkeit immer seltener von der Sprache Gebrauch machen und schließ. lich ganz von derselben Abstand nehmen. Diesen ungeheuren Sprung soll die Fortbildungsschule verkleinern; sie soll die Kinder von der Taubstummenanstalt in das Leben hinüberführen.

$\mathrm{Zu}$ diesem $\mathrm{Zwecke}$ hat der sprachliche Unterricht in den Fortbildungsschulen in der Weise eine Erweiterung zu erfahren,

1) Rundschau, Blätter für Taubstummenbildung, $\mathrm{XX}$. Jhrg. No. 9. 1. Mai 1907. 
daß derselbe nicht nur von Taubstummenlehrern, wie Gutzmann will, sondern auch von Volkssehullehrern und den der Fortbildungsschule zur Sonderausbildung zu überweisenden, jungen Geistlichen erteilt wird. Bei der Verteilung des Unterrichts auf die 3 Kategorien von Lehrern soll im 1. und 2. Fortbildungskursus der Taubstummenlehrer; im 3. und 4. Kursus der Geistliche und Volksschullehrer die Mehrzahl der Stunden erteilen.

So würden die taubstummen Kinder mehr und mebr geübt, auch mit anderen Personen als nur den Taubstummenlehrern sprachlich zu. verkehren und würden besser gerüstet als bisher in das Leben hinaustreten.

An die Fortbildungsschulen sind dieTa ta s tu m m en-Leh rerbildungsanstalten anzuschließen.

Ich glaube, dab die Durchführung dieser Vorschläge das Taubstummenbildungswesen erheblich fördern würde. Nach der heutigen Organisation enthalten die Taubstummenanstalten nicht ganz wenige Kinder, die nicht nur keinen nenmenswerten Nutzen von dem Unterricht ziehen, sondern denselben sogar nicht ganz selten stören; sie bleiben in den Anstalten, weil man nicht weib, wo man sie unterbringen soll. Ich glaube, die Taubstummenlehrer würden nichts dagegen einzuwenden haben, wenn man diese Kinder als Gruppe I aussonderte and in einer besonderen Anstalt unterbrächte.

Ebenso dürfte von ihrer Seite kein Einspruch dagegen zu erheben sein, daß man die besonders begabten, taubstummen Kinder aus einem gröberen Bezirk in einer Taubstummenanstalt vereinigt.

Es könnte nun scheinen, als ob zu der vorgeschlagenen Reorganisation des Taubstummenbildungswesens ganz besonders grobe Mittel erforderlich wären. Dies dürfte keineswegs der Fall sein, wie eine kurze Überlegung ergibt. Es bedarf nur des Zusammenschlusses zweier oder mehrerer Provinzen zu gemeinsamer Arbeit auf diesem Gebiet.

In einer Provinz finden sich kaum soviel Kinder, um eine, wenn auch nur kleine Anstalt von 30 bis 40 Kindern der I. und III. Gruppe zu bilden; denn die Hauptmasse der Taubstummen gehört in die II. Gruppe. Beim Zusammenschluß von 2 oder selbst 3 Provinzen würde sich dagegen meiner Schätzung: nach sehr wohl je eine kleinere Anstalt der I. und III. Gruppe zusammenstellen lassen.

Die ganze zu lösende Aufgabe würde also darin bestehen, daß einerseits jede Provinz für sich an 1 oder 2 der bestehenden 
Taubstummenanstalten eine zweiklassige Vorschule angliedert und andererseits mit einer oder zwei benachbarten Provinzen ein Übereinkommen dahin trifft, daßs eine Anstalt alle in ihren Bezirken zur Gruppe I. und eine zweite Anstalt alle zur Gruppe III. gehörenden Kinder aufnimmt. Alle Kinder der Gruppe II. verbleiben in der Fürsorge ibrer Heimatprovinz, wie auch die $\mathrm{Er}$ richtung der Fortbildungsschulen Sache jeder einzelnen Provinz ist. Neue Taubstummenanstalten wïrden von jeder Provinz nur insoweit zu errichten sein, als bisher für eine Anzahl der taubstummen Kinder die Möglichkeit der Beschulung überhaupt feblte.

Diese ev. neu za errichtenden Taubstummenanstalten müssen Externate und nicht Internate sein und in kleinen Landstädten mit vorwiegend Acker- und Gartenbau treibender Bevölkerung errichtet werden; denn nach meinen Erfahrungen wirken der ständige Verkehr mit vollsinnigen Personen, die mannigfache Gelegenheit zur Mitarbeit im Feld und Garten, die einfachen Verhältnisse der kleinen Stadt auf die geistige und körperliche Entwicklung der Kinder ungemein viel günstiger, als wenn sie in Internaten von der Außenwelt abgeschlossen und wesentlich auf den Verkehr untereinander angewiesen sind.

Es möchte scheinen, als ob uns die vorstehenden Aasfïhrungen $\mathrm{zu}$ einer nicht nur wünschenswerten, sondern $\mathrm{m}$. E. dringend notwendigen Reorganisation des Taubstummenbildungswesens weit ab von der fürsorgenden Tätigkeit des Arztes für die taubstummen Kinder geführt habe. Dies ist jedoch nur scheinbar der Fall; denn diese Vorschläge sind ja unter dem Gesichtspunkte der fürsorgenden Tätigkeit des Arztes für die Sprachentwicklung, Spracherhaltung und das Sprachverständnis der taubstummen Kinder gemacht.

Die fürsorgende Tätigkeit des Arztes hat sich aber nicht allein hierauf $\mathbf{z u}$ erstrecken, sondern weiter auf die Beseitigung von Krankheitszuständen überbaupt wie insbesondere solcher des Ohres, des Auges, der Nase, des Rachens wie des Kehlkopfes und auf die Gesunderhaltung dieser Organe. Die Spezialärzte, Augen- wie Ohrenärzte, sind indes nur relativ selten ständige Berater in den Taubstummenanstalten; nur bei der Untersuchung der neu aufgenommenen Kinder werden sie in nicht wenigen Anstalten hinzugezogen. Der laufende ärzliche Dienst liegt dem Anstaltsarzt ob, nach dessen Entscheidung, wie es scheint, in der Mehrzahl der Anstalten die Hilfe des Spezialarztes nachgesucht wird. 
Eine ministerielle Umfrage vom 7. September 1898 ergab „daß nur in seltenen Fällen die spezialärztliche Fürsorge dem Bedürfnis der Anstalten und dem gegenwärtigen Stande der medizinisehen Wissenschaft entspricht" - Ministerielle Verfügung vom 23. April 1900 -, und fithrte zur Errichtung besonderer, fakultativer Unterrichtskurse für die Hausärzte über Taubstummenanstalten an der Königlichen Taubstummenanstalt zu Berlin. Die Untersuchung und Behandlung des Ohres, des Rachens, des Kehlkopfes wie des Auges bildeten neben der Physiologie, Psychologie und Pathologie der Sprache wie neben Vorträgen über Taubstummenbildung die hauptsächlichsten Unterrichtsgegenstände.

Hoffen wir, daß unsere Bemühungen auf dem Gebiete der ärztlichen Fürsorge für die taubstummen Kinder weiter auf fruchtbaren Boden fallen, und daß die hier gemachten Vorschläge einen neuen Anstoß geben zur Fortentwicklung des Taubstummenbildungswesens. 